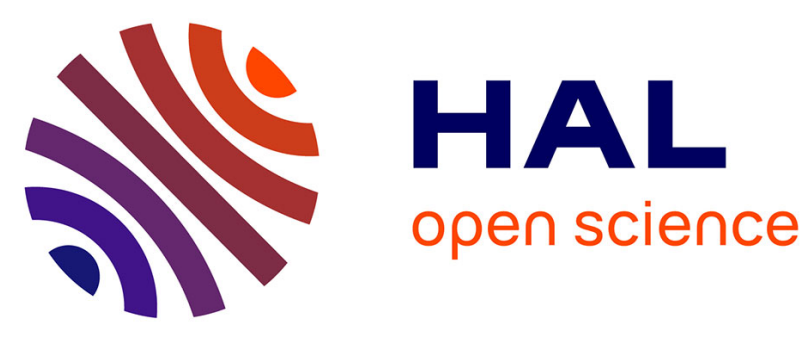

\title{
Towards a predictive simulation of brace action in adolescent idiopathic scoliosis
}

Claudio Vergari, Zhuowei Chen, Léopold Robichon, Isabelle Courtois, Eric

Ebermeyer, Raphaël Vialle, Tristan Langlais, Raphaël Pietton, Wafa Skalli

\section{- To cite this version:}

Claudio Vergari, Zhuowei Chen, Léopold Robichon, Isabelle Courtois, Eric Ebermeyer, et al.. Towards a predictive simulation of brace action in adolescent idiopathic scoliosis. Computer Methods in Biomechanics and Biomedical Engineering, In press, 10.1080/10255842.2020.1856373 . hal-03156362

\section{HAL Id: hal-03156362 \\ https://hal.science/hal-03156362}

Submitted on 2 Mar 2021

HAL is a multi-disciplinary open access archive for the deposit and dissemination of scientific research documents, whether they are published or not. The documents may come from teaching and research institutions in France or abroad, or from public or private research centers.
L'archive ouverte pluridisciplinaire $\mathbf{H A L}$, est destinée au dépôt et à la diffusion de documents scientifiques de niveau recherche, publiés ou non, émanant des établissements d'enseignement et de recherche français ou étrangers, des laboratoires publics ou privés. 


\title{
Towards a predictive simulation of brace action in adolescent idiopathic scoliosis
}

\author{
Claudio Vergari ${ }^{\mathrm{a}} \mathbb{D}$, Zhuowei Chen ${ }^{\mathrm{a}}$, Léopold Robichon ${ }^{\mathrm{a}}$, Isabelle Courtois ${ }^{\mathrm{b}}$, Eric Ebermeyer ${ }^{\mathrm{b}}$, Raphaël \\ Vialle $^{c}$, Tristan Langlais ${ }^{c}$, Raphaël Pietton ${ }^{c}$ and Wafa Skalli ${ }^{a}$ \\ ${ }^{a}$ Arts et Métiers Institute of Technology, Université Sorbonne Paris Nord, IBHGC - Institut de Biomécanique Humaine Georges \\ Charpak, HESAM Université, F-75013, Paris; ${ }^{\mathrm{b}} \mathrm{CHU}$ - Hôpital Bellevue, Unité Rachis, Saint-Etienne, France; ' ${ }^{\mathrm{D}}$ epartment of Pediatric \\ Orthopaedics, Sorbonne Université, Hôpital Armand Trousseau, Assistance \\ Publique-Hôpitaux de Paris (AP-HP), Paris, France
}

\begin{abstract}
Bracing is the most common treatment to stop the progression of adolescent idiopathic scoliosis. Finite element modeling could help improve brace design, but model validation is still a challenge. In this work, the clinical relevance of a predictive and subject-specific model for bracing was evaluated in forty-six AIS patients. The model reproduces brace action and the patient's spinopelvic adjustments to keep balance. The model simulated $70 \%$ or more patients with geometrical parameters within a preselected tolerance level. Although the model simulation of the sagittal plane could be improved, the approach is promising for a realistic and predictive simulation of brace action.
\end{abstract}

\section{KEYWORDS}

Spine deformity; orthosis; finite element modelling; biomechanics

\section{Introduction}

Adolescent idiopathic scoliosis (AIS) is a $3 \mathrm{D}$ deformity of the spine and the trunk; it can be progressive, and if the progression is left unchecked, it can lead to pulmonary or locomotor impairment. The main treatment to stop the progression early is bracing along with physiotherapeutic scoliosis-specific exercises (Negrini et al. 2018).

Long-term efficacy of bracing strongly depends on patient compliance (Weinstein et al. 2013), but efficacy can only be evaluated at the end of the treatment, when the patient's outcome is either a progression of the deformity requiring fusion surgery, or a negligible progression when skeletal maturity is reached. Several factors can help predict brace efficacy early, such as wear time, closed triradiate cartilage and low body mass index, although the best predictor of long-term efficacy seems to be the short term inbrace correction, which can be quantified via radiological exam (Upadhyay et al. 1995; Clin et al. 2010; van den Bogaart et al. 2019). However, obtaining a good in-brace correction is not straightforward: while braces can often correct Cobb angle, the correction of the deformity in the axial plane and the restoration of the sagittal curves is still a challenge (Courvoisier et al. 2013).

Indeed, planning and fabrication of braces is often based on the experience of the orthotist. Finite element modeling (FEM) of the spine and rib cage appears to be a promising approach (Nie et al. 2008; Berteau et al. 2011; Desbiens-Blais et al. 2012; Wang et al. 2014; Cobetto et al. 2016; Vergari et al. 2016; Courvoisier et al. 2019). However, validation of these approaches is still usually limited to the coronal plane, and no assessment on spinal axial rotation or rib hump is performed.

A framework to comprehensively evaluate the clinical relevance of a FEM for brace simulation was proposed and tested on a series of 42 patients (Vergari et al. 2015, 2016). The method is based on a comparison of measured and simulated values for a selection of geometrical parameters which are used in clinical routine to describe the trunk deformity (Table 1 ). The parameters were chosen to assess the model in $3 \mathrm{D}$.

However, it is still unclear how to take into account the compensation mechanisms deployed by the patient to keep balance in-brace (Vergari et al. 2020). This limitation was underlined in Vergari et al. (2016), where the in-brace pelvic tilt and the position

CONTACT Claudio Vergari c.vergari@gmail.com $\Theta$ Arts et Métiers ParisTech, Institut de Biomécanique Humaine Georges Charpak, 151 bd de I'Hôpital, 75013 Paris, France 
Table 1. Geometrical parameters (VAR: vertebral axial rotation at the apex). Tolerance values are calculated as indicated in (Vergari et al. 2015).

\begin{tabular}{lcccc}
\hline & Before brace & In-brace & Simulation & Tolerance \\
\hline T1-T12 kyphosis $\left[^{\circ}\right]$ & $35.2 \pm 15.5$ & $30.6 \pm 14.1$ & $33.4 \pm 15.2$ & 7.8 \\
T4-T12 kyphosis $\left[^{\circ}\right]$ & $23.4 \pm 15.7$ & $18.8 \pm 14.2$ & $23.3 \pm 14.6$ & 5.4 \\
L1-L5 lordosis $\left[^{\circ}\right]$ & $47.5 \pm 13.9$ & $41.4 \pm 10.6$ & $46.3 \pm 11.8$ & 6.5 \\
Cobb angle $\left[^{\circ}\right]$ & $27.4 \pm 11.8$ & $18.3 \pm 9.5$ & $17.2 \pm 10.7$ & 4.4 \\
VAR $\left[{ }^{\circ}\right]$ & $9.7 \pm 6.4$ & $7.7 \pm 5.6$ & $7.9 \pm 5.8$ & 4.8 \\
Torsion $\left[^{\circ}\right]$ & $7.8 \pm 4.7$ & $7 \pm 5.5$ & $8 \pm 5.1$ & 5.7 \\
Rib hump $\left[^{\circ}\right]$ & $8.2 \pm 3.7$ & $7.8 \pm 3.1$ & $10.1 \pm 4.5$ & 7.1 \\
Pelvic tilt $\left[{ }^{\circ}\right]$ & $5.5 \pm 7.4$ & $7.1 \pm 5.8$ & $6.6 \pm 5.7$ & 4.8 \\
\hline
\end{tabular}

of $\mathrm{T} 1$ relative to the pelvis were imposed in the model as boundary conditions.

The hypothesis of the present work was that such predictive relationships between pre-brace and inbrace alignment can be used to drive a FEM of the trunk for the simulation of brace action in AIS. This hypothesis was then tested by simulating the brace action in forty-six AIS patients at their first brace wear and by comparing the clinical parameters with the simulated data.

\section{Methods}

\section{Subjects}

Forty-six patients were included (average age: $13 \pm 2,38$ girls and 8 boys, average Cobb angle $27.4 \pm 11.8^{\circ}$ ). All patients were diagnosed with AIS: according to the Lenke classification system, twenty-six patients were Lenke 1, thirteen Lenke 3 and eight were Lenke 5 (Lenke et al. 2001). All patients were prescribed a brace; 34 wore thoracic lumbar sacral orthosis (TLSO) and 12 wore night-time braces. TLSOs are usually worn during the day and they aim to reducing the main scoliotic curve, while restoring the sagittal curves. Night-time braces aim to hypercorrecting the curve.

Patients were excluded if the cause of their scoliosis was known (non-idiopathic cases) or if they underwent previous orthotic treatment. Biplanar radiographs (EOS System, EOS Imaging, Paris, France) were acquired when the decision of treatment was reached, and later at the first brace wear. The delay between the two acquisitions was $3 \pm 2$ months. Data collection was approved by the ethical committee (CPP 6001 Ile de France V), and patients and their parents signed an informed consent.

\section{Subject-specific finite element model}

The spine (T1-L5), ribcage and pelvis of each patient was reconstructed in $3 \mathrm{D}$ with previously validated methods (Humbert et al. 2009; Ghostine et al. 2017;
Vergari et al. 2020), ; both before and in-brace (Figure 1). This reconstruction allowed automatic calculation of $3 \mathrm{D}$ geometrical spinopelvic parameters, but it also provided $3 \mathrm{D}$ models of all vertebrae, pelvis and rib cage, that were used to build a subject-specific finite element model of the trunk (Figure 1). The model was previously described in detail (Vergari et al. 2016); it implements the thoracolumbar spine, including surface contacts of articular facets and nonlinear mechanical properties of intervertebral discs and ligaments. The ribs inertial properties were based on an existing database of scoliotic adolescent ribs morphology (Sandoz et al. 2013), while their overall geometry was patient-specific and their mechanical properties were adapted to the patient's Risser sign (Pezowicz and Głowacki 2012).

The model was built both for the before and inbrace configurations in a common anatomic reference frame based on the pelvis. A robust coordinate system was also associated to the T1 vertebra (Humbert et al. 2009), which allowed calculating the $3 \mathrm{D}$ position and orientation in both configurations. Pelvic tilt was also computed as the angle in the sagittal plane between the line connecting the middle of the interacetabular axis with center of the sacral plate, and the vertical.

\section{Boundary conditions}

Two classes of boundary conditions were applied: those aiming to reproduce the effect of the brace on the patient's thorax, and those aiming to predict the patient's balance as altered by the brace.

To reproduce the correcting effect of the brace, pressure points were identified on the frontal and lateral radiographs by observing local deformations of the outer soft tissues (where the brace pads apply pressure) and the mechanical radiopaque elements of the brace. This action was simulated using soft pads (Figure 1, 485 nodes, 433 hexahedral elements, $0.01 \mathrm{MPa}$ Young's modulus) acting on the rib cage through mechanical contact or, for those pads acting on the lumbar region or posteriorly on the spine, by displacing the corresponding vertebra. Displacements of the pad or of the vertebra were computed by comparing the out of brace and in-brace $3 \mathrm{D}$ reconstructions.

Patient balance was predicted through the analysis of balance compensation mechanisms between prebrace and in brace geometrical parameters (Vergari et al. 2020). It was observed that the variation induced by the brace on the sagittal and axial orientation of T1 vertebra and on the sagittal orientation of the pelvis (i.e., pelvic tilt) could be predicted from 

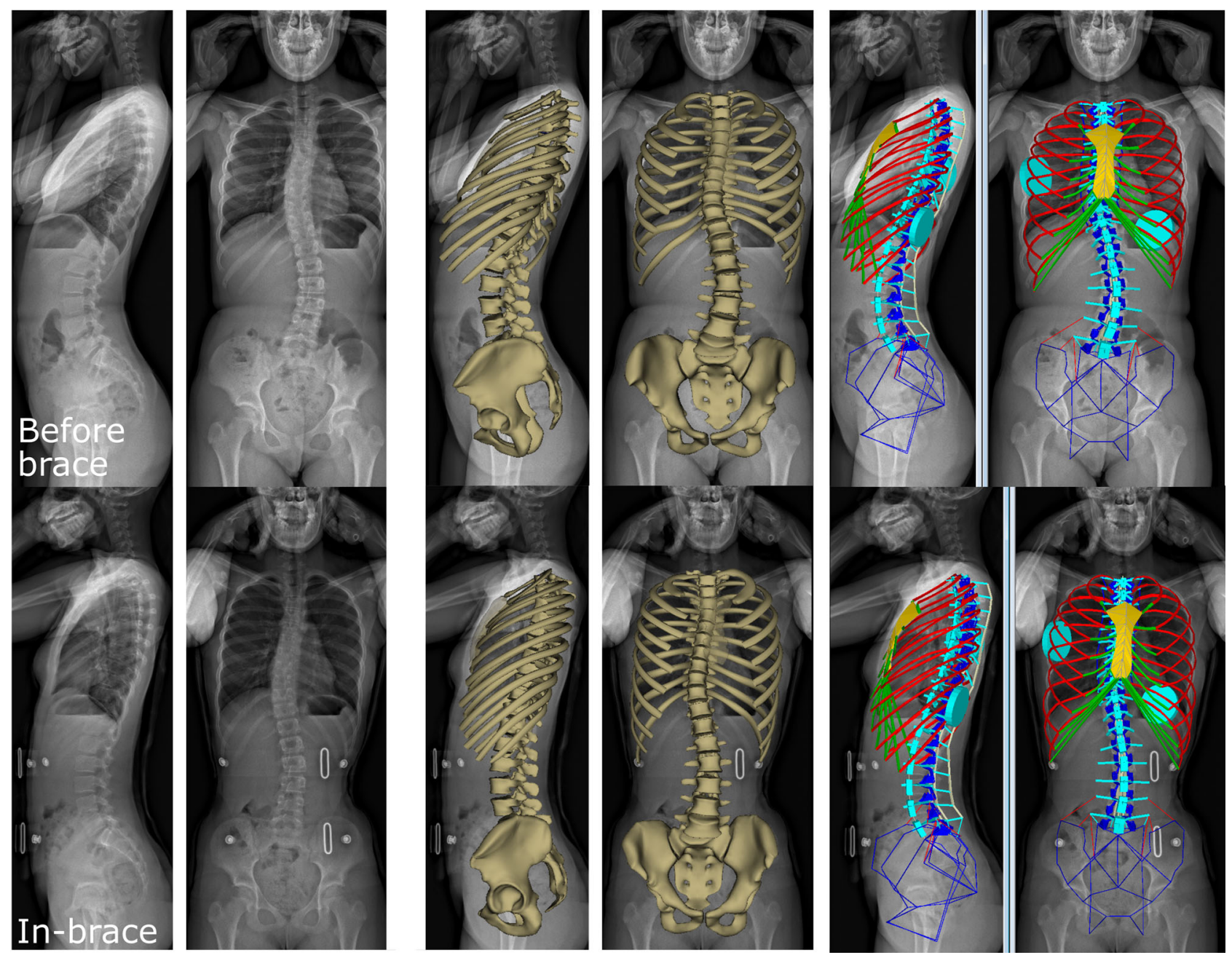

Figure 1. Biplanar x-ray, 3D reconstruction and finite element model of the spine, pelvis and ribcage before (top row) and inbrace (bottom row)).

pre-brace values (Figure 2). Similarly, the change of position of $\mathrm{T} 1$ relative to the pelvis could be estimated from pre-brace values. To reduce overfitting, the regression models between pre-brace parameters and their in-brace changes were calculated with a leave-one-out method.

These relationships were applied as boundary conditions to $\mathrm{T} 1$ and the pelvis, i.e., a sagittal rotation was imposed to the pelvis to simulate the change of pelvic tilt and the predicted rotations and displacements were applied to T1. However, in order to leave some freedom to the model in reacting to the brace pad actions, these conditions were not applied directly to the anatomical structures, but through linear and rotational springs attached to $\mathrm{T} 1$ vertebral body and to the pelvic acetabula. Therefore, the applied boundary conditions did not force the anatomical structure towards its predicted pose, but rather softly pushed them there. The stiffness of these springs was selected through trial and error.
The model was implemented in Ansys $2020 \mathrm{R} 1$ (Ansys Inc., Canonsburg, PA, USA) while all analyses were performed in Matlab 2018b (The Mathworks Inc, Natick, MA, USA).

\section{Model evaluation}

The clinical significance of the model was evaluated using a previously proposed framework, based on the comparison of simulated and measured clinical geometrical parameters (Vergari et al. 2015), which are listed in Table 1 . Tolerance values $(\varepsilon)$ were calculated for each parameter as $\varepsilon=\sqrt{2\left(2 R M S_{S D}\right)^{2}}$, where $2 \mathrm{RMS}_{\mathrm{SD}}$ is the uncertainty due to the reconstruction method which was previously estimated (Humbert et al. 2009). Thus, this tolerance approximates the minimal error that can be detected when comparing two $3 \mathrm{D}$ reconstructions; differences between simulated and measured parameters below this threshold 

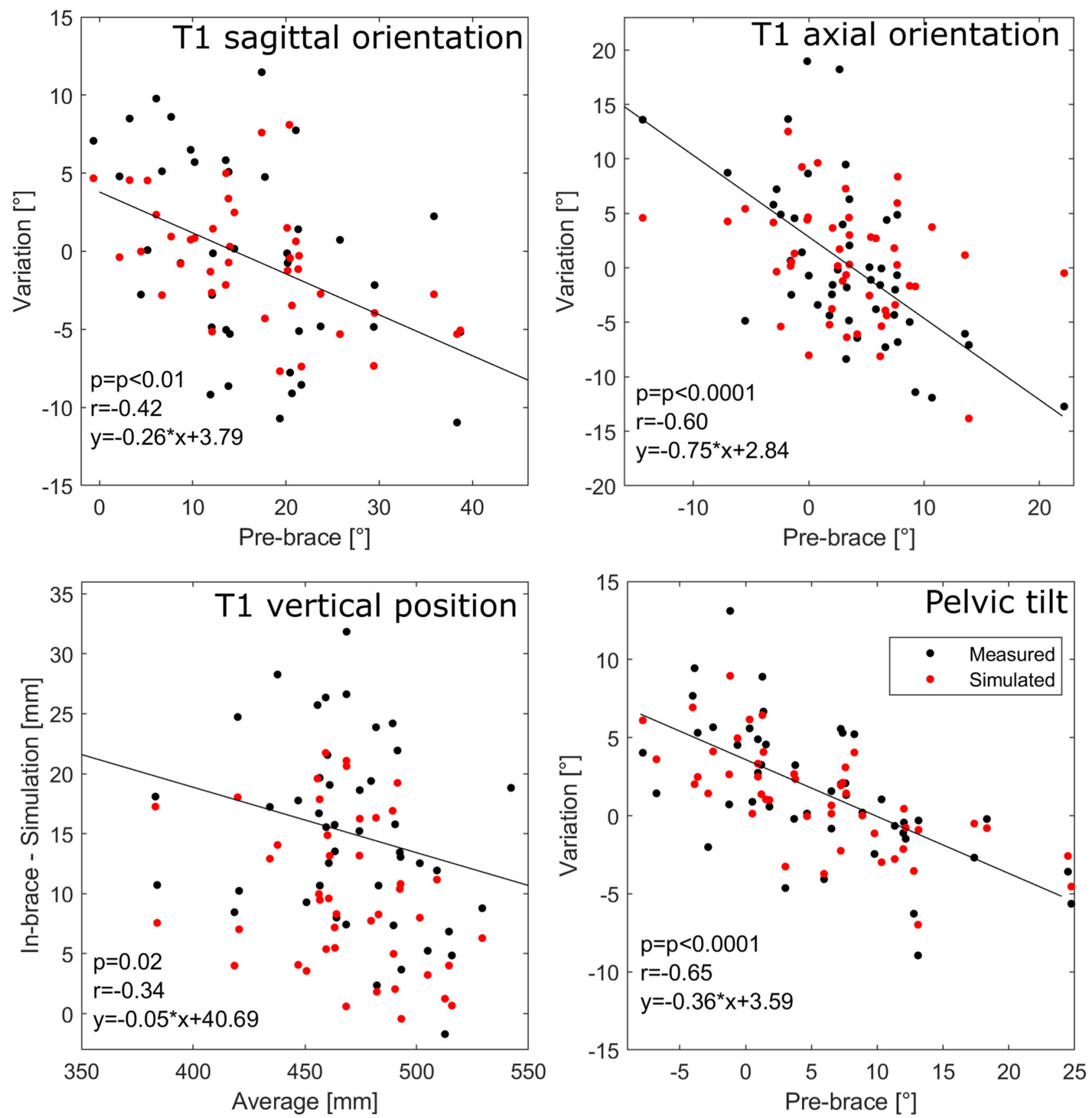

Figure 2. In-brace variation of T1 vertebra sagittal orientation, axial orientation, vertical position, and variation of pelvic tilt as a function of pre-brace values. Both measured and simulated values are reported.

were considered acceptable. Finally, simulated and measured values were compared with Bland-Altmann plots. Values were compared with paired t-tests (normality was checked with Lilliefors test) and independent $\mathrm{t}$-tests when comparing brace types.

\section{Results}

Brace measured and simulated effects were reported in Table 1. Overall, the brace had a good correction of Cobb angle (from $27.4 \pm 11.8^{\circ}$ to $18.3 \pm 9.5^{\circ}$, $\mathrm{p}=0.0001$ ), but it also had a flattening effect on the thoracolumbar spine (decrease of kyphosis and lordosis); the decrease in L1-L5 lordosis was significant $(p=0.02)$. Braces also left the axial plane almost unchanged $\left(2^{\circ}\right.$ reduction of VAR and $0.8^{\circ}$ reduction of torsion, $\mathrm{p}>0.05)$.

The brace effect measured after the simulation was similar, although the simulation tended to reduce the flattening of the back (Table 1). The only significant difference between simulated and in-brace values concerned the L1-L5 lordosis $(\mathrm{p}=0.04)$. Furthermore, no 


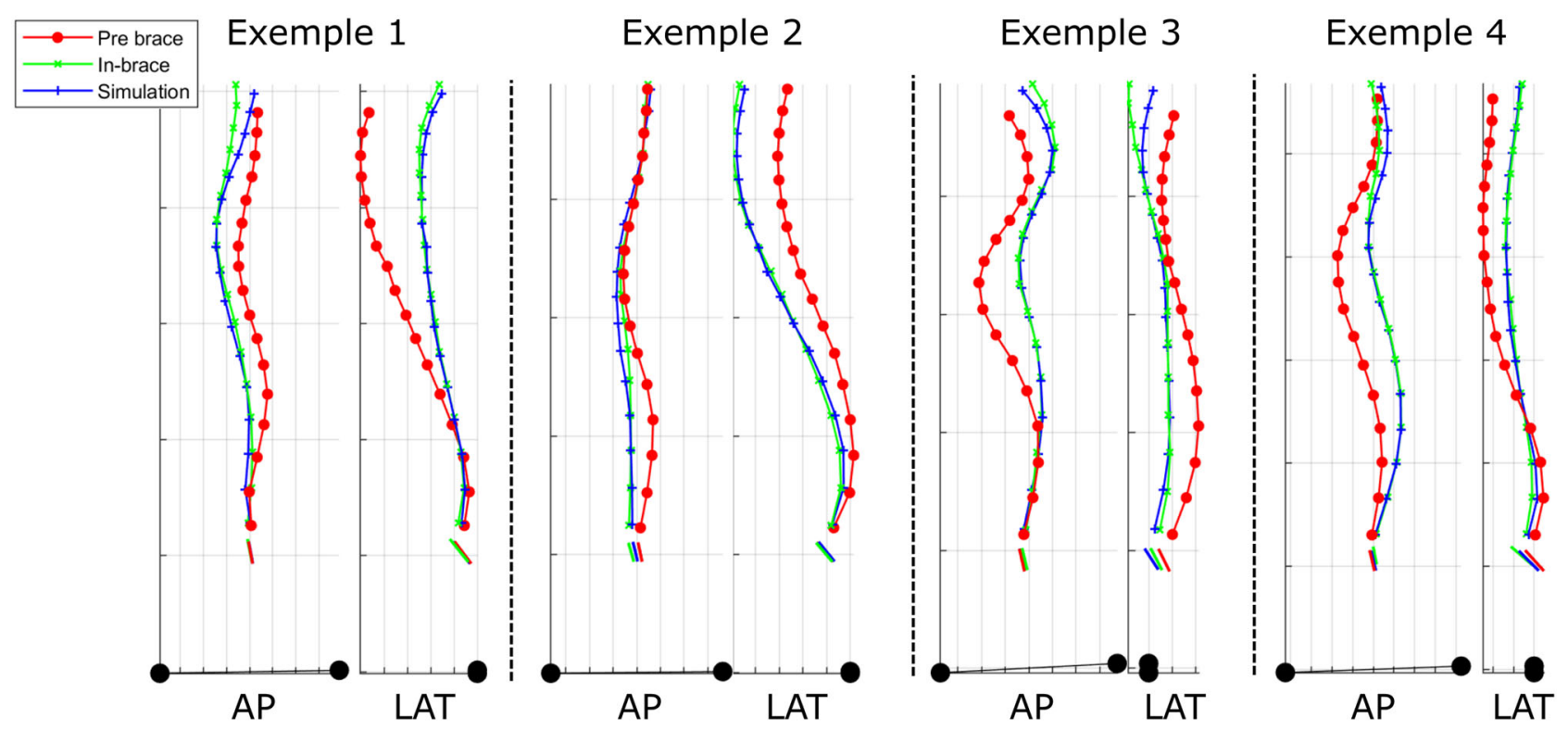

Figure 3. Representative spine midlines pre brace, in-brace and after simulation; the sacral endplate is also represented in the three conditions, while the inter-acetabular axis is represented in black, since it is the same in the all conditions. Examples 1 and 2 are patients wearing thoracic lumbar sacral orthosis, while examples 3 and 4 wore night-time braces.

significant difference was observed when comparing absolute errors in the simulation of TLSOs or nighttime braces $(\mathrm{p}>0.05)$.

Prediction of $\mathrm{T} 1$ and pelvis movements are reported in Figure 2, in which the measured values and the results obtained in the simulation are compared. They do not correspond exactly because these values were imposed as boundary conditions through passive springs; hence, the model could "resist" the imposed displacements. T1 sagittal and axial orientation could be predicted with an R-value $>0.4$, while the uncertainty of the vertical position was slightly higher $(r=0.34)$. The average vertical displacement of T1 was $14.9 \pm 7.5 \mathrm{~mm}$, whilst its average change of orientation in the sagittal, coronal, and axial planes were $0.2 \pm 6.8^{\circ}, 0.7 \pm 5.1^{\circ}$ and $0.3 \pm 7.3^{\circ}$, respectively.

Figure 3 shows four representative examples of spinal midlines pre-brace, in-brace and resulting from the simulation. Examples are shown for both TLSOs and night-time braces.

Bland-Altmann plots comparing measured and simulated clinical parameters are reported in Figure 4. The bias was lower than $5^{\circ}$ for all parameters. Figure 5 represents the number of patients below the tolerance levels for each parameter; results are compared with those of a non-predictive version of this model which was published previously (Vergari et al. 2016). $70 \%$ or more patients were below the tolerance level in all parameters but for T4-T12 kyphosis, for which only $54 \%$ of the patients were below tolerance.
Average simulation time was 37 seconds, and once the $3 \mathrm{D}$ reconstruction was performed, and the pads were positioned, the process of building the model and postprocessing was fully automatic.

\section{Discussion}

In this work, a predictive model of brace action on the scoliotic trunk was compared to experimental data to assess the clinical relevance of the model, while considering the three-dimensional character of the deformity. Furthermore, tolerance values were calculated for each parameter, balancing the uncertainty due to the comparison of $3 \mathrm{D}$ reconstructions while remaining consistent with minimal clinically relevant variations. The present version of the FEM is truly predictive since it does not require in-brace information to simulate brace action. For validation purposes, the model was used to reproduce the action of existing braces in order to have a gold standard to make a comparison. However, already at this stage the model can be used to test alternative corrective actions on the trunk to optimize its action.

One of the most challenging issues in the use of FEMs in vivo corrective treatment is the lack of understanding of the postural adaptations that occur in-brace, which depend on the patient's neuromusculoskeletal control. In recent years, an invariant was described, which could drive this postural control: the head tends to keep above the pelvis in wide range of 

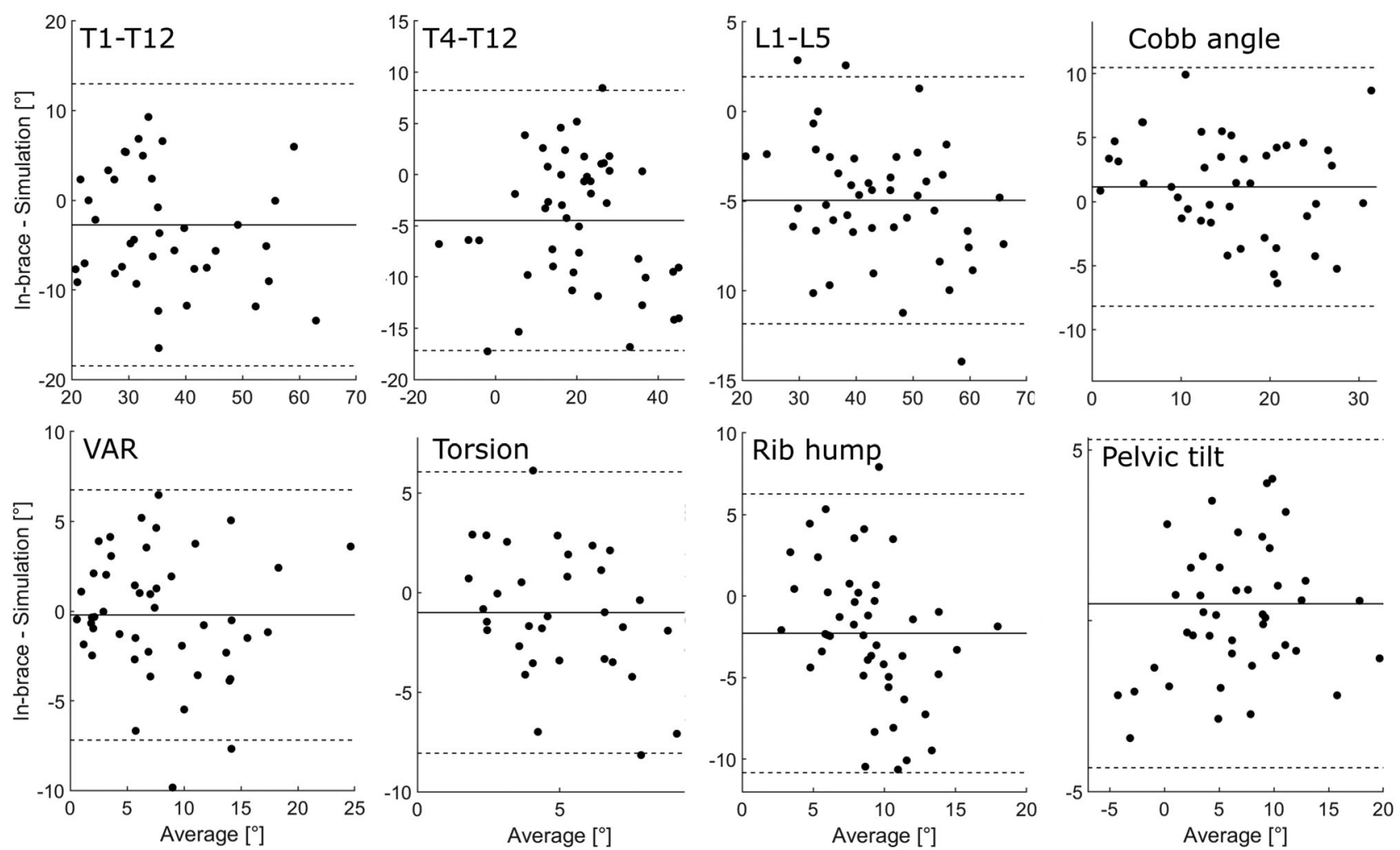

Figure 4. Bland-Altmann plots comparing the measured and simulated spinopelvic geometrical parameters. VAR I the vertebral axial rotation at the apical level. These parameters represent the simulation in the three planes of space (kyphosis and pelvic tilt: sagittal plane; Cobb angle: coronal plane; Torsion, VAR, rib hump: axial plane).

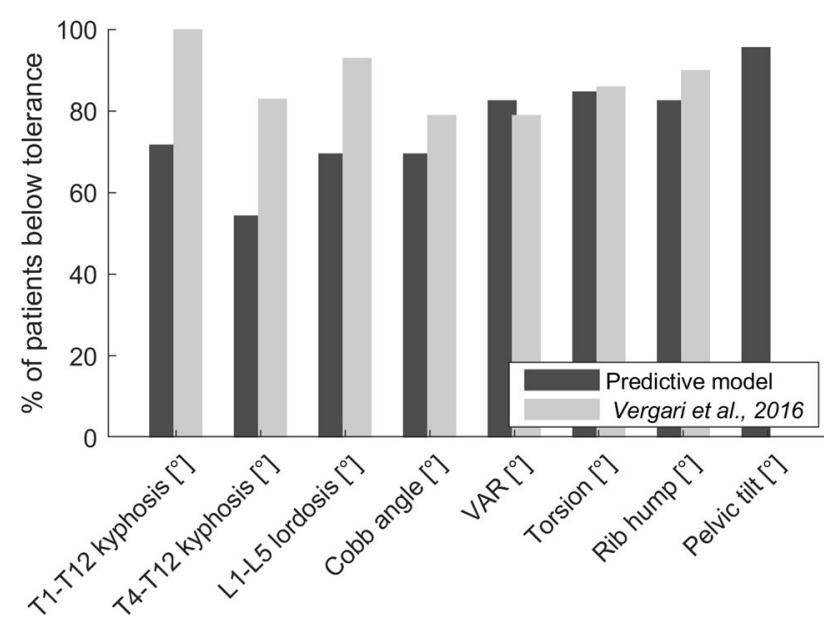

Figure 5. Percentage of patients below the tolerance difference for each geometrical parameter in the present predictive model and in (Vergari et al. 2016); pelvic tilt is missing in the latter because it was imposed as boundary condition. Tolerance values are reported in.

subjects and situations. During respiratory-related postural perturbations (Attali et al. 2019), before and after scoliosis surgery (Alzakri et al. 2019) and, more importantly, in and out of brace (Vergari et al. 2020). In order to maintain this invariant, schemes of postural adaptation are progressively being explored, and pelvic reorientation seems to play a key role in this adaptation. This is the reason why predicting pelvic reorientation was considered important. This FEM is the first that implements such postural adaptation, albeit in a very preliminary approach.

The model still presents some limitations. For instance, it is passive, and the reaction of the simulated trunk to the external loads is mainly driven by the skeletal geometry and by the mechanical properties. This is a common limitation of most existing models, given the difficulty of including muscle action and motor control in these simulations. Still, the implemented prediction of in-brace spinopelvic alignment is a preliminary step towards a full neuromusculoskeletal model, including central motor control and muscular action.

The second limitation is that different types of braces were included (TLSOs and night-time), and their action was simulated through pads rather than a full brace. Although the biomechanical principle of most braces is similar, night-time braces aim to hyper-correcting the scoliotic curve by lateral bending towards curve convexity (Violas et al. 2009). Furthermore, night-time braces are designed to be worn when the patient is supine, but here brace action was assessed with standing radiographs. Methods exist to account for the changes from standing to supine position in 
numerical models (Dumas et al. 2005; Lafon et al. 2009; Clin et al. 2010), but this was beyond the scope of this paper which aimed to comparing FEM results with clinical parameters which were measured in standing patients. These two brace type seemed to induce a similar pattern of spinopelvic realignment to TLSOs (Vergari et al. 2020), and the simulation does not seem to be affected by brace type or by the hypercorrection of night-time braces (Figure 3).

Finally, the same dataset was used to estimate the relationships to predict the spinopelvic alignment and to validate the model; however, a leave-one-out procedure was used to mitigate the effects of overfitting.

The main innovation of the present version of the FEM is the use of predictive models to simulate inbrace spinopelvic alignment. This approach seems promising, although the simulation of the back-flattening effect of the brace could be improved. Indeed, the comparison in Figure 5 shows that the improvement of the model from non-predictive (Vergari et al. 2016) to predictive did not significantly deteriorate the model's accuracy, apart from kyphosis and lordosis. This result could be expected as in the previous iteration the position and orientation of $\mathrm{T} 1$ was imposed as a boundary condition using information from the in-brace $3 \mathrm{D}$ reconstruction, while now this parameter is predicted.

Prediction of in-brace pelvic tilt seemed to reproduce well the patient's pelvic realignment, since 95\% of patients were below the tolerance for this parameter (Figure 5). Figure 2 shows that pelvic tilt undergoes changes up to $10^{\circ}$ or $-10^{\circ}$ when the patient is wearing a brace. Hence, this pelvic reconfiguration cannot be neglected in the simulation since this will directly affect the patient's sagittal alignment.

Prediction of T1 vertical position was less accurate, with an R-value of $-0.34(\mathrm{p}=0.02$, Figure 5), which helps to explain the difference in kyphosis between the model and simulation. Surprisingly, normalized values of $\mathrm{T} 1$ vertical position, for instance relative to interacetabular distance, did not yield stronger correlations. The average value for the change of $\mathrm{T} 1$ vertical position was $14.9 \pm 7.5 \mathrm{~mm}$. Interestingly, the correlation between the patient's initial height and inbrace vertical growth was negative, indicating that taller patients tended to grow slightly less. A patient with a vertical distance between $\mathrm{T} 1$ and the interacetabular axis of $350 \mathrm{~mm}$ tends to grow by $22 \mathrm{~mm}$ inbrace, while a patient presenting an initial distance of 550 would only grow by $11 \mathrm{~mm}$.

The correlation between pre- and in-brace $\mathrm{T} 1$ axial orientation was also negative (Figure 2). A value of zero for this orientation indicates that $\mathrm{T} 1$ is oriented towards the front of the patient, while non-zero values correspond to right or left horizontal orientations. Therefore, Figure 5 shows that patients tended to correct their $\mathrm{T} 1$ axial orientation towards zero: patients with a negative value pre-brace showed a positive variation, and vice versa. Similarly, the symmetrical variation of $\mathrm{T} 1$ sagittal orientation (Figure 2) suggests that patients with extreme values tended to change this orientation towards the average value of $14 \pm 12^{\circ}$.

\section{Conclusion}

Models for the $3 \mathrm{D}$ analysis of spinal deformity and their response to different brace designs should be assessed with extensive clinical validation studies before they can be applied for clinical practice. A predictive and subject-specific finite element model to simulate brace action was described in the present work. The model allowed the reproduction of the three-dimensional effect in different braces on the scoliotic trunk and, whilst the underlying predictive model can be improved for the sagittal plane. This can already be used to simulate alternative brace configurations to improve short-term efficacy.

\section{Acknowledgments}

The authors are grateful to the ParisTech BiomecAM chair program on subject-specific musculoskeletal modelling (with the support of ParisTech and Yves Cotrel Foundations, Proteor, Société Générale and Covea). We are also thankful to David Barrie Colridge for his support.

\section{Disclosure statement}

Dr. Skalli has a patent related to biplanar X-Rays and associated $3 \mathrm{D}$ reconstruction methods, with no personal financial benefit (royalties rewarded for research and education) licensed to EOS Imaging. Dr. Vialle reports personal fees and grants (unrelated to this study) from EOS Imaging. This study was partly funded by the company Proteor.

\section{Ethics approval}

The data collection was approved of by the ethical committee (CPP 6001 Ile de France V), and patients and their parents signed an informed consent.

\section{ORCID}

Claudio Vergari (D) http://orcid.org/0000-0002-7049-2405 


\section{References}

Alzakri A, Vergari C, Van den Abbeele M, Gille O, Skalli W, Obeid I. 2019. Global sagittal alignment and proximal junctional kyphosis in adolescent idiopathic scoliosis. Spine Deform. 7(2):236-244.

Attali V, Clavel L, Rouch P, Rivals I, Rémy-Néris S, Skalli W, Sandoz B, Similowski T. 2019. Compensation of respiratory-related postural perturbation is achieved by maintenance of head-to-pelvis alignment in healthy humans. Front Physiol. 10:441.

Berteau J-P, Pithioux M, Mesure S, Bollini G, Chabrand P. 2011. Beyond the classic correction system: a numerical nonrigid approach to the scoliosis brace. Spine J. 11(5): 424-431.

Clin J, Aubin C-E, Parent S, Labelle H. 2010. A biomechanical study of the Charleston brace for the treatment of scoliosis. Spine. 35(19):E940-7. http://www.ncbi.nlm.nih. gov/pubmed/20431434.

Cobetto N, Aubin CE, Parent S, Clin J, Barchi S, Turgeon I, Labelle H. 2016. Effectiveness of braces designed using computer-aided design and manufacturing (CAD/CAM) and finite element simulation compared to CAD/CAM only for the conservative treatment of adolescent idiopathic scoliosis: a prospective randomized controlled trial. Eur Spine J. 25(10):3056-3064. http://link.springer. com/10.1007/s00586-016-4434-3.

Courvoisier A, Drevelle X, Vialle R, Dubousset J, Skalli W. 2013. 3D analysis of brace treatment in idiopathic scoliosis. Eur Spine J. 22(11):2449-2455. http://dx.doi.org/10. 1007/s00586-013-2881-7.

Courvoisier A, Nesme M, Gerbelot J, Moreau-Gaudry A, Faure F. 2019. Prediction of brace effect in scoliotic patients: blinded evaluation of a novel brace simulatoran observational cross-sectional study. Eur Spine J. 28(6): 1277-1285..

Desbiens-Blais F, Clin J, Parent S, Labelle H, Aubin C-E. 2012. New brace design combining CAD/CAM and biomechanical simulation for the treatment of adolescent idiopathic scoliosis. Clin Biomech. 27(10):999-1005. http://linkinghub.elsevier.com/retrieve/pii/ S0268003312001805.

Dumas R, Lafage V, Lafon Y, Steib JP, Mitton D, Skalli W. 2005. Finite element simulation of spinal deformities correction by in situ contouring technique. Comput Methods Biomech Biomed Eng. 8(5):331-337. https:// www.ncbi.nlm.nih.gov/entrez/query.fcgi? $\mathrm{cmd}=$ Retrieve $\&$ $\mathrm{db}=$ PubMed\&dopt=Citation\&list_uids $=16298855$.

Ghostine B, Sauret C, Assi A, Bakouny Z, Khalil N, Skalli W, Ghanem I. 2017. Influence of patient axial malpositioning on the trueness and precision of pelvic parameters obtained from $3 \mathrm{D}$ reconstructions based on biplanar radiographs. Eur Radiol. 27(3):1295-1302. httpss://doi. org/10.1007/s00330-016-4452-x.

Humbert L, De Guise JA, Aubert B, Godbout B, Skalli W. 2009. 3D reconstruction of the spine from biplanar Xrays using parametric models based on transversal and longitudinal inferences. Med Eng Phys. 31(6):681-687. https://www.ncbi.nlm.nih.gov/pubmed/19230743.

Lafon Y, Lafage V, Dubousset J, Skalli W. 2009. Intraoperative thraee-dimensional correction during rod rotation technique. Spine. 34(5):512-519. http://www. ncbi.nlm.nih.gov/pubmed/19247171.

Lenke LG, Betz RR, Harms J, Bridwell KH, Clements DH, Lowe TG, Blanke K. 2001. Adolescent idiopathic scoliosis: a new classification to determine extent of spinal arthrodesis. J Bone Joint Surg Am. 83(8): 1169-1181.

Negrini S, Donzelli S, Aulisa AG, Czaprowski D, Schreiber S, de Mauroy JC, Diers H, Grivas TB, Knott P, Kotwicki T, et al. 2018. 2016 SOSORT guidelines: orthopaedic and rehabilitation treatment of idiopathic scoliosis during growth. Scoliosis Spinal Disord. 13(1):3.

Nie W-Z, Ye M, Wang Z-Y. 2008. Infinite models in scoliosis: a review of the literature and analysis of personal experience. Biomed Tech. 53(4):174-180.

Pezowicz C, Głowacki M. 2012. The mechanical properties of human ribs in young adult. Acta Bioeng Biomech. 14(2):53-60.

Sandoz B, Badina A, Laporte S, Lambot K, Mitton D, Skalli W. 2013. Quantitative geometric analysis of rib, costal cartilage and sternum from childhood to teenagehood. Med Biol Eng Comput. 51(9):971-979. https://dx.doi. org/10.1007/s11517-013-1070-5.

Upadhyay SS, Nelson IW, E K W, L C S, Leong JCY. 1995. New Prognostic Factors to Predict the Final Outcome of Brace Treatment in Adolescent Idiopathic Scoliosis. Spine (Phila Pa 1976). 20(5). https://doi.org/10.1097/ 00007632-199503010-00006

van den Bogaart $M$, van Royen BJ, Haanstra TM, de Kleuver M, Faraj SSA. 2019. Predictive factors for brace treatment outcome in adolescent idiopathic scoliosis: a best-evidence synthesis. Eur Spine J [Internet]. (0123456789). https://doi.org/10.1007/s00586018-05870-6

Vergari C, Aubert B, Lallemant-Dudek P, Haen TX, Skalli W. 2020. A novel method of anatomical landmark selection for rib cage $3 \mathrm{D}$ reconstruction from biplanar radiography. Comput Methods Biomech Biomed Eng Imaging Vis. 8(1):15-23. https://doi.org/10.1080/21681163.2018. 1537860.

Vergari C, Courtois I, Ebermeyer E, Bouloussa H, Vialle R, Skalli W. 2016. Experimental validation of a patient-specific model of orthotic action in adolescent idiopathic scoliosis. Eur Spine J. 25(10):3049-3055. https://dx.doi. org/10.1007/s00586-016-4511-7.

Vergari C, Courtois I, Ebermeyer E, Pietton R, Bouloussa H, Vialle R, Skalli W. 2019. Head to pelvis alignment of adolescent idiopathic scoliosis patients both in and out of brace. Eur Spine J. 28(6):1286-1295. https://doi.org/ 10.1007/s00586-019-05981-8.

Vergari C, Ribes G, Aubert B, Adam C, Miladi L, Ilharreborde B, Abelin-Genevois K, Rouch P, Skalli W. 2015. Evaluation of a patient-specific finite-element model to simulate conservative treatment in adolescent idiopathic scoliosis. Spine Deform. 3(1):4-11. https://doi. org/10.1016/j.jspd.2014.06.014.

Violas P, Colobert B, Vervaecke D, Dufournet JJ, Rakotomanana L. 2009. Objective measurement of biomechanics effects of brace use for adolescent idiopathic scoliosis: a comparison between standing and supine 
position. Comput Methods Biomech Biomed Eng. 12(sup1):251-252..

Wang W, Baran GR, Betz RR, Samdani AF, Pahys JM, Cahill PJ. 2014. The use of finite element models to assist understanding and treatment for scoliosis: a review paper. Spine Deform. 2(1):10-
27. http://www.sciencedirect.com/science/article/pii/S22 12134 X13001366.

Weinstein SL, Dolan LA, Wright JG, Dobbs MB. 2013. Effects of bracing in adolescents with idiopathic scoliosis. N Engl J Med. 369(16):1512-1521. [Internet]http://www. nejm.org/doi/full/10.1056/NEJMoa1307337. 